\title{
Usage of Light Emitting Diodes (LEDs) for improved satellite tracking
}

Paolo Marziolia ${ }^{\mathrm{1}}$, Andrea Gianfermo ${ }^{\mathrm{a}}$, Lorenzo Frezzaa, Diego Amadio ${ }^{\mathrm{a}}$, Niccolò Picci ${ }^{\mathrm{a}}$, Federico Curianò ${ }^{a}$ Maria Giulia Pancallia, Eleonora Vestito ${ }^{a}$, Justin Schachter ${ }^{b}$, Matt Szczerbab ${ }^{b}$ Daniel Gu ${ }^{b}$, Anny Lin ${ }^{b}$, James Cutler ${ }^{\mathrm{b}}$, Simone Pirrotta ${ }^{\mathrm{c}}$, Fabio Santoni ${ }^{\mathrm{d}}$, Patrick Seitzer ${ }^{\mathrm{e}}$, Fabrizio Piergentilia

${ }^{a}$ Department of Mechanical and Aerospace Engineering, Sapienza University of Rome, via Eudossiana 18, 00184 Rome, Italy

${ }^{b}$ Department of Aerospace Engineering, University of Michigan, 1320 Beal Ave, Ann Arbor, MI 48109

c Italian Space Agency (ASI), via del Politecnico, 00133 Rome, Italy

${ }^{d}$ Department of Astronautics, Electric and Energy Engineering, Sapienza University of Rome, via Eudossiana 18, 00184 Rome, Italy

${ }^{e}$ Department of Astronomy, University of Michigan, 1085 S. University, Ann Arbor, MI 48109

\begin{abstract}
With the increasing number of satellite launches, especially in Low Earth Orbit (LEO), optical tracking can offer a convenient enhancement of tracking precision and availability. Spaceborne active illumination devices, such as LED payloads, can offer a significant improvement to optical observations, extending the observability interval to the whole eclipse time and performing optimized flash sequences for identification, orbit determination, attitude reconstruction or low data rate communication. The main features of LED panels for optical tracking mounted on small satellites platforms (and with particular regards to nano-satellite platforms) are outlined in this paper, along with the description of the design drivers. The analysis of the performance is referred to Sun-Synchronous (at $700 \mathrm{~km}$ of altitude) and International Space Station $(400 \mathrm{~km})$ orbits, while the ground segment and the optical link budget reference design relies on a standard university space debris observation station architecture. The paper also outlines the advantages of using different observation techniques and the variety of flashing patterns. The LEDSAT $1 U$ CubeSat, aiming at demonstrating the effectiveness of an LED-based payload for observation and tracking, is used as a study case for examples of the LED payloads and related operations that are reported and described in this paper.
\end{abstract}

Keywords: LED, satellites, CubeSat, optical, tracking, identification

\footnotetext{
${ }^{1}$ Corresponding author.

Address: DIMA - Dept. of Mechanical and Aerospace Engineering

via Eudossiana 18, 00184 Rome (RM), Italy

e-mail address: paolo.marzioli@uniroma1.it
} 


\section{Introduction}

The increase of satellite launches, especially in the framework of an increasingly numerous deployment of small satellites (classifiable as nano-satellites when the mass is lower than $10 \mathrm{~kg}$, microsatellites if between 10 and $100 \mathrm{~kg}$, minisatellites if between 100 and $500 \mathrm{~kg}$ ) in Low Earth Orbit (LEO), stresses the need for more reliable, precise and punctual spacecraft tracking. The required improvement in tracking and measurement availability can be understood in the perspective of new generation instrumentation for Space Traffic Management (STM) [1-4].

While the Space Situational Awareness (SSA) operations are usually guaranteed by the exploitation of radars, optical tracking can support the surveillance tasks and improve the accuracy of the Two-Line Elements (TLE) estimation for tracked objects [5]. The availability of optical measurements shows an obvious correlation with weather and lighting conditions, since the observation site, besides being influenced by good weather, shall be in darkness while the target object is in sunlight [5].The inherently passive nature of the measurements to be acquired allows one to relax the requirements for the spacecraft tracking equipment, resulting in relatively low-cost instrumentation and data acquisition architectures [6]. This enhances the possibility of building observational networks all over the world at low cost and with a high measurements availability [7].

The spacecraft optical trackability can be significantly improved by implementing an active source of illumination on the external surfaces of the satellite. LEDs (Light Emitting Diodes) can be a simple solution to relax or remove the aforementioned constraint of data acquisition on lighting conditions, since VIS-band (Visible band) diodes can be observed throughout the orbital eclipse phase [8-10]. LEDs can be commanded to perform specific sequences for enhancing the spacecraft tracking during all mission phases.

For example, at deployment, an early identification of small satellites is difficult when performed through radar systems, especially when the spacecraft is deployed within large clusters. For the first weeks upon deployment, the identity of satellites belonging to the same cluster might be confused, leading to a reduced awareness on the satellite behavior in LEOP (Launch and Early Operations). This might be potentially critical when telecommands are needed for continuing the nominal satellite operations. During the orbital lifetime, LEDs can be utilized for improving orbit determination accuracy, as well as for enabling or enhancing attitude determination [11]. When implementing LED boards on multiple sides of the spacecraft and commanding them to flash with different patterns, the satellite's sides pointing towards Earth can be recognized and tracked by optical observatories. Finally, a spacecraft can use LEDs as basic data downlink system by encoding data on the flashes. Orbital data, housekeeping and status information can be downlinked to ground-based observatories in case of a main RF communication system failure (which occurs, in the only case of CubeSats, in the $20 \%$ of mission failure cases [12]), temporary outage or even in case of a pointing error of directional transmitting antenna towards Earth [13].

LED payloads have already been studied for on-ground communications, establishing data links on maritime vessels [14] or for other on-ground Free Space Optics (FSO) applications for ensuring connectivity in large environments [15] or for positioning purposes [16]. In the aerospace field, LED detectors have been designed for balloon applications as light pollution sensors [17]. For what concerns satellite applications, LED payloads have been applied for intra-satellite optical links $[18,19]$ and inter-satellite communication by micro-satellite platforms [20,21]. While laser data links are able to provide much higher data rates [22-24] or novel applications for enhancing the communications security [25-28], but by presenting strict pointing requirements, LEDs offer a wide angle of emission able to make short-range inter-satellite communications more flexible to various attitude states of satellite swarms [20]. Furthermore, the observability of LEDs on- 
board a nano-satellite platform from ground-based optical sensors has already been verified with the FITSAT-1 nano-satellite in 2012 [29]. The application of such space-to-ground optical link to observations for tracking, attitude determination and back-up communication will be verified by LEDSAT. This 1-Unit CubeSat (approximately $100 \times 100 \times 110 \mathrm{~mm}$ ) is equipped with one LED board mounted on each side and it aims at demonstrating the effectiveness of LED boards for enhanced trackability of small satellites platforms. The satellite has been conceived in 2016 at the Sapienza Space Systems and Space Surveillance Laboratory (S5Lab) at Sapienza University of Rome, in collaboration with the University of Michigan. The LEDSAT development has begun in 2017, after being accepted for the European Space Agency (ESA) Fly Your Satellite! Programme. The spacecraft is now under development at Sapienza University of Rome. LEDSAT is also part of the Italian Space Agency (ASI) IKUNS (Italian-Kenyan University Nano-Satellites) Programme, as it has given to Kenyan students the opportunity to participate in all the phases of the CubeSat development. In the meantime, the Michigan eXploration Laboratory (MXL) team from University of Michigan was able to prove the long range observability of LED payloads during stratospheric balloon tests over a noisy city environment.

This paper describes the opportunities offered by LED-based boards for the spacecraft tracking, early recognition, attitude reconstruction and back-up communication. The typical design for VIS-band LED boards is considered, providing an overview of observability, consumed power and interfaces of those devices, confirming the easy integrability of such systems into nanosatellite platforms. The operations scheduling and design will be presented for improved early recognition, orbit determination, attitude determination, light-based communication and post-mission disposal, reporting the preferred patterns outline and the optimal ground segment configuration for each task. The paper will use LEDSAT as a study case, in order to provide applied features and data to a real space mission scenario.

\section{LED-based tracking system design drivers and requirements}

The LED-based tracking is comprised of an active space segment and a passive ground segment. The spacecraft will be covered on each face by a different colored LED board which will be controlled to flash with specific patterns at pre-determined times. An on ground optical observational station will observe the LED flashes to acquire measurements. The LED power and the ground segment sensitivity shall be coupled in order to assure visibility of the satellite in a sufficiently large range of elevation angles. The system's major design driver should aim at maximizing of the LEDs irradiance in order to include smaller optical observatories in the ground segment. A higher number of observatories, constituting a distributed network architecture, can lead to a significant improvement in the precision of the acquired optical data [7].

\subsection{Space segment: LED panels features}

The number of LEDs to mount on each panel can usually shift from 4 to 40 , depending on the implemented diode models. The number is mainly influenced by the peak power exerted by the spacecraft Electrical Power System (EPS), which usually stays around $30 \mathrm{~W}$ for nano-satellite platforms. In the case of LEDSAT, a number between 20 and 30 diodes is mounted on each board ( 20 diodes on blue and green boards, 30 diodes on red boards), respecting the peak power limit of $30 \mathrm{~W}$. The different number of diodes reflects the different irradiance provided by the LED models selected for the implementation on the spacecraft. Wide emission angles are preferred in order to ease the acquisition of data from a wider range of locations and observatories, without any concerns on the attitude of the satellite. A higher Quantum Efficiency (QE) is obviously preferred to reduce the power consumption for a fixed radiated flux value. 
To ensure the survivability of the diodes in space, an environmental testing campaign shall be conducted on test LED boards. The qualification campaign should consider at least the following tests:

- Total Ionizing Dose (TID) and Displacement Damage (DD) radiation testing, for ensuring the diodes to be compliant with the mission time in the simulated orbit environment (LEDSAT was qualified to radiation to TID testing in 2017 [30]);

- Ultra-Violet (UV) radiation testing, for ensuring that the LED wavelengths does not deviate towards the yellow color due to yellowing of the silicone cover of the LED ([30]);

- Thermal-Vacuum qualification in both unbiased and biased conditions, to confirm the survivability of the LEDs for external mount on satellites;

- Vibration testing for the prototype LED boards to verify the survivability of assembly and solder joints;

- Outgassing test and characterization of the materials to comply with launch authority requirements.

For the interfaces, the most critical matching between the LED system and the satellite bus is usually represented by the electrical interface. LED boards with an irradiance higher than $10 \mathrm{~W}$ shall take into account the maximum power delivery of the small satellite power system. The LED duty cycles shall be also matched to the mission nature, in order to reduce the energy drain from the batteries and to lower the severity of the satellite Depth of Discharge (DoD). A brief analysis on example duty cycles has been run when applying VIS-BAND LEDs (active in umbra) as secondary payloads. By considering as tentative orbit cases the ISS (International Space Station) orbit and a SSO (Sun-Synchronous Orbit) at $700 \mathrm{~km}$ with LTAN (Local Time of Ascending Node) 12.00, the generated power by a $1 \mathrm{U}$ CubeSat is taken into account as worst case scenario. The target of the calculation is to derive the maximum flash time for an example pattern by considering a maximum energy consumption of $5 \%$ the average generated power in one orbit. The budget considers the execution of example " $\mathrm{R}$ " Morse letters [8]. The pattern considers a total period of $1.6 \mathrm{~s}$ and a flash time of $0.3 \mathrm{~s}$ per pattern. The flash pattern time graph is reported in Figure 1 . The budget is reported in Table 1.

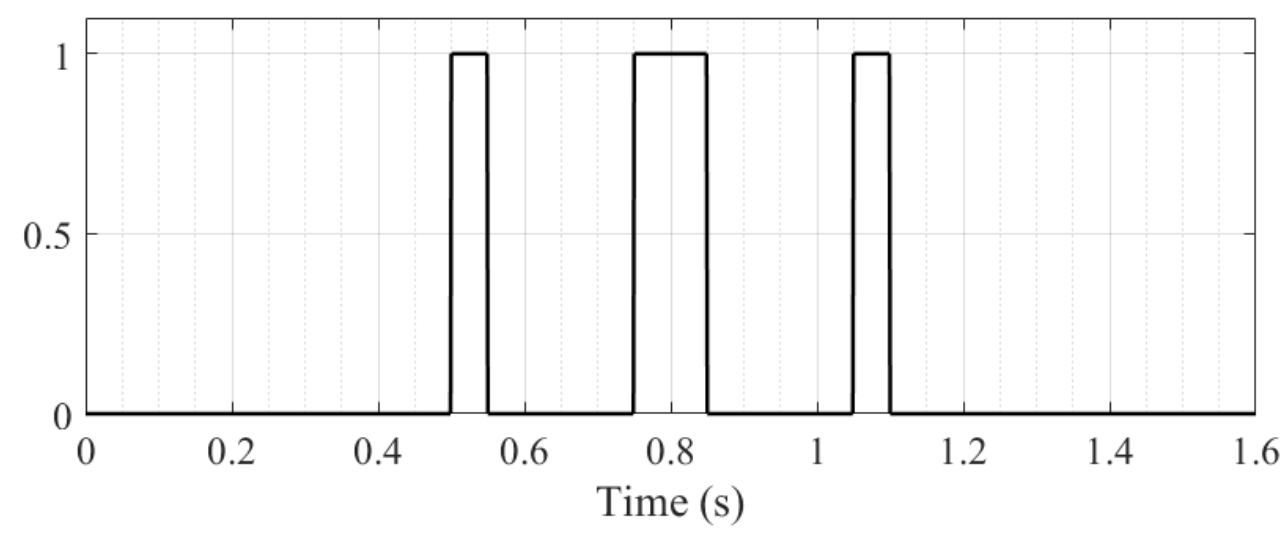

Figure 1: Example " $R$ " Morse letter pattern.

Table 1: Flashing time and patterns execution period for example 1 U CubeSats in ISS and SSO.

\begin{tabular}{|l|l|l|}
\hline & ISS orbit (400 km) & SSO orbit, 700 km, 12.00 LTAN \\
\hline $\begin{array}{l}\text { Average generated power (1U } \\
\text { CubeSat) }\end{array}$ & $\mathbf{2}$ W \\
\hline Sunlight time (per orbit) & $3794 \mathrm{~s}$ & $3816 \mathrm{~s}$ \\
\hline Total generated energy & $2.108 \mathrm{Whr}$ & $2.120 \mathrm{Whr}$ \\
\hline
\end{tabular}




\begin{tabular}{|l|l|l|}
\hline $\begin{array}{l}\text { Available energy to LED payload } \\
\text { (5\% of the total generated } \\
\text { energy) }\end{array}$ & $\mathbf{0 . 1 0 5} \mathbf{~ W h r}$ & $\mathbf{0 . 1 0 6} \mathbf{W h r}$ \\
\hline $\begin{array}{l}\text { Example LED peak power } \\
\text { consumption }\end{array}$ & $25.00 \mathrm{~W}$ & $25.00 \mathrm{~W}$ \\
\hline Available flashing time & $15.18 \mathrm{~s}$ & $15.26 \mathrm{~s}$ \\
\hline Umbra average time & $1937 \mathrm{~s}$ & $2098 \mathrm{~s}$ \\
\hline $\begin{array}{l}\text { LED duty cycle (over umbra } \\
\text { time) }\end{array}$ & $\mathbf{0 . 7 3 \%}$ & $\mathbf{0 . 7 8 \%}$ \\
\hline $\begin{array}{l}\text { Required flashing time for } \\
\text { example "K" letter pattern }\end{array}$ & $0.2 \mathrm{~s}$ & $0.2 \mathrm{~s}$ \\
\hline $\begin{array}{l}\text { Available pattern repetitions per } \\
\text { eclipse phase }\end{array}$ & $\mathbf{7 5}$ & $\mathbf{7 6}$ \\
\hline $\begin{array}{l}\text { Repetition time of pattern for } \\
\text { each umbra phase }\end{array}$ & $\mathbf{2 5 . 8 3 ~ \mathrm { s }}$ & $\mathbf{2 7 . 6 0 \mathrm { s }}$ \\
\hline
\end{tabular}

The LED flashes can be aligned with time reference signals. For example, the LED flashing commands can be aligned to the GPS (Global Positioning System) PPS (Pulse-per-Second) signal to ensure time synchronization with the GPS UTC (Universal Coordinated Time), and/or use a fine timekeeping device (such as a crystal oscillator or a miniaturized atomic clock) to match the flashes to the satellite operations [11].

The mechanical fixture for fastening the boards to the satellite external faces can be evaluated case by case, mainly depending on the size of the orbital platform. In general, LED boards containing up to 40 LEDs can be accommodated on 1-Unit CubeSat faces. In the LEDSAT case, boards are fastened to the CubeSat main structure through the solar panels screws. A picture of the assembled satellite, with the LED boards fastened on the solar panel, is provided in Figure 2.

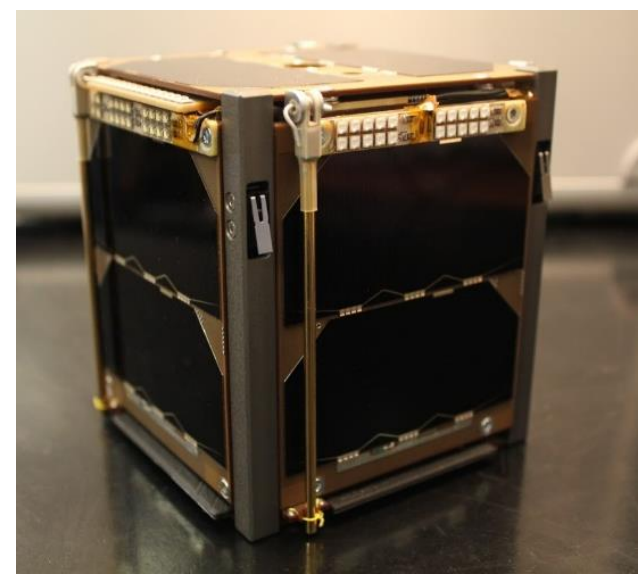

Figure 2: the LEDSAT $1 U$ CubeSat. The LED boards on the solar panels are visible in the picture.

Control electronics shall be included for controlling the LED flashes, either assembled on the same board or on a small controller board to place inside the satellite. The size of the control electronics is compatible with all the nano-, micro- and small satellite platforms and do not impose large constraints for the implementation of LED payloads on such platforms. 


\subsection{Ground Segment: Optical observatories architecture}

The ground segment design should take into account reliable, low-cost, hardware. University space debris observatories, typically with an overall hardware on the order of tens of thousands of dollars, can be implemented for the LED boards observation. A typical configuration of an optical observatory in VIS-band band is reported, as part of the Sapienza ground stations network that will be used for LEDSAT [6,31].

Table 2: Typical configuration of optical observatory for observing spaceborne LEDs.

\begin{tabular}{|c|c|c|c|}
\hline Component & Feature & \multicolumn{2}{|l|}{ Value } \\
\hline Optical Tube & Focal Length & \multicolumn{2}{|l|}{$1500 \mathrm{~mm}$} \\
\hline & Aperture & \multicolumn{2}{|l|}{$250 \mathrm{~mm}$} \\
\hline & Field of View (FOV) & \multicolumn{2}{|l|}{2 degrees } \\
\hline Electronic Mount & Go-to Speed & \multicolumn{2}{|l|}{$>3$ degrees $/ \mathrm{s}$} \\
\hline \multirow[t]{3}{*}{$\begin{array}{l}\text { Implementable sensor } \\
\text { and preferred function }\end{array}$} & $\begin{array}{l}\text { CCD } \\
\text { (Charge-Coupled Device) }\end{array}$ & $\begin{array}{l}\text { Sidereal - orbit } \\
\text { determination and } \\
\text { attitude } \\
\text { reconstruction }\end{array}$ & $\begin{array}{l}\text { High } \\
\text { sensitivity- } \\
\text { very low } \\
\text { acquisition } \\
\text { speed }\end{array}$ \\
\hline & $\begin{array}{l}\text { CMOS } \\
\text { (Composite Metal Oxide } \\
\text { Semiconductor) }\end{array}$ & $\begin{array}{l}\text { Sidereal and } \\
\text { tracking-orbit } \\
\text { determination, } \\
\text { attitude } \\
\text { reconstruction and } \\
\text { optical } \\
\text { communication }\end{array}$ & $\begin{array}{l}\text { High } \\
\text { sensitivity - } \\
\text { medium } \\
\text { acquisition } \\
\text { speed - } \\
\text { versatility }\end{array}$ \\
\hline & APD/p-i-n photodiodes & $\begin{array}{l}\text { Tracking - optical } \\
\text { communication }\end{array}$ & $\begin{array}{l}\text { Low } \\
\text { sensitivity- } \\
\text { High } \\
\text { acquisition } \\
\text { speed }\end{array}$ \\
\hline Filters & \multicolumn{3}{|c|}{$\begin{array}{l}\text { Implementable if needing to increase SNR (Signal-to-Noise } \\
\text { Ratio). Filter wheels are be applicable to multi-color LEDs } \\
\text { observations }\end{array}$} \\
\hline \multirow[t]{3}{*}{ Available Functionalities } & \multicolumn{3}{|l|}{ Automated Tracking } \\
\hline & \multicolumn{3}{|l|}{ Remote controllability } \\
\hline & \multicolumn{3}{|l|}{ Operations Scheduling [32] } \\
\hline
\end{tabular}

The observational techniques to be considered for the LED data acquisition are the following:

- Sidereal tracking, exploits the telescope motion at sidereal rate to maintain the stellar background field fixed in the FOV while the satellite pass appears as light trails. The pointing position can be retrieved from the satellite (or launcher vehicle) TLE or a best guess for the satellite pass. While the generally small telescope FOV allows for a shorter observation interval, the technique presents a higher operational reliability, not relying on the precision of the mount go-to command during the passage, but maintaining the telescope pointed towards a fixed celestial coordinate position.

- Target tracking, exploited by commanding the telescope to follow the satellite trajectory. This can be retrieved from the satellite estimated TLEs. The technique allows for a higher retrievable data amount during a single passage, while its exploitation depends on the precision of the TLEs and on the accuracy of the motorized telescope pointing command system. When using this technique, videos are shot to the satellite pass, which appears as a dot when stars move through the sensor FOV. 
CCD sensors are mainly applicable to sidereal tracking, as long-exposure pictures of a pass are more meaningful, while CMOS sensors can be applied to both the pass typologies. Photodiodes (either APD, Avalanche Photodiodes or $\mathrm{p}-\mathrm{i}-\mathrm{n}$ ) have preferred usage on target tracking, since the data is originated by the photodiode voltage of the observed target over time and they cannot be applicable to sidereal tracking mode.

If implementing LEDs in different colors (as for the LEDSAT mission), simultaneous observations from close locations can be implemented with color filters in order to evaluate the radiometric data in different wavelengths and a color recognition of the faces if different colors are used on each of them.

\subsection{Optical link budget and apparent magnitude}

The definition of the features of the space and ground segment allows to calculate the optical link budget for ensuring the LED boards visibility during the mission.

The link budget can be calculated in analogy with RF link budget estimations for data communication with satellites. The establishment and performance estimation of an optical link results more difficult than a conventional RF link due for a higher constraint on line of sight and enhanced limitations on atmospheric absorption. Therefore, a link budget analysis of the optical link is needed to characterize the space and ground segment and the interaction with the medium to obtain the link achievability.

The main parameters that resemble a RF link budget are the free space loss and the behavior in transmission of the LEDs that operate like an antenna for calculation of the equivalent isotropic radiated power. The main parameters of the link budget, specified for the telescope station architecture outlined in paragraph 2.2, are summarized in Table 3.

Table 3: Optical link budget main parameters [13]

\begin{tabular}{lcc}
\hline Parameter & Value & Unit \\
Wavelength & 660 & $\mathrm{~nm}$ \\
$\begin{array}{l}\text { Transmitted optical } \\
\text { power }\end{array}$ & 12.5 & $\mathrm{~W}$ \\
LED Emission Angle & 150 & $\mathrm{deg}$ \\
$\begin{array}{l}\text { Zenith Atmospheric } \\
\text { Transmittance }\end{array}$ & 0.7 & - \\
$\begin{array}{l}\text { Optics focal length } \\
\text { Optics aperture }\end{array}$ & 1.5 & $\mathrm{~m}$ \\
Pixel size & 25 & $\mathrm{~cm}$ \\
\hline
\end{tabular}

The optical parameter that particularly affects the link is atmospheric absorption. This varies in the visible spectrum and can be simplified by calculating the air masses included in the Line of Sight path. The air mass is used prevalently in astronomy to calculate the extinction of the light in the atmosphere and a unit of air mass represents the quantity of air that the observer sees at his zenith (or at $90^{\circ}$ of elevation). Air masses 
increase exponentially at lower elevation angle therefore observation under 20 degrees are very difficult, as demonstrated by the estimation of the air masses at different elevation angles shown in Figure 3a. Atmospheric transmittance for a zenith observation in relation to the wavelength is shown in Figure $3 \mathrm{~b}$.

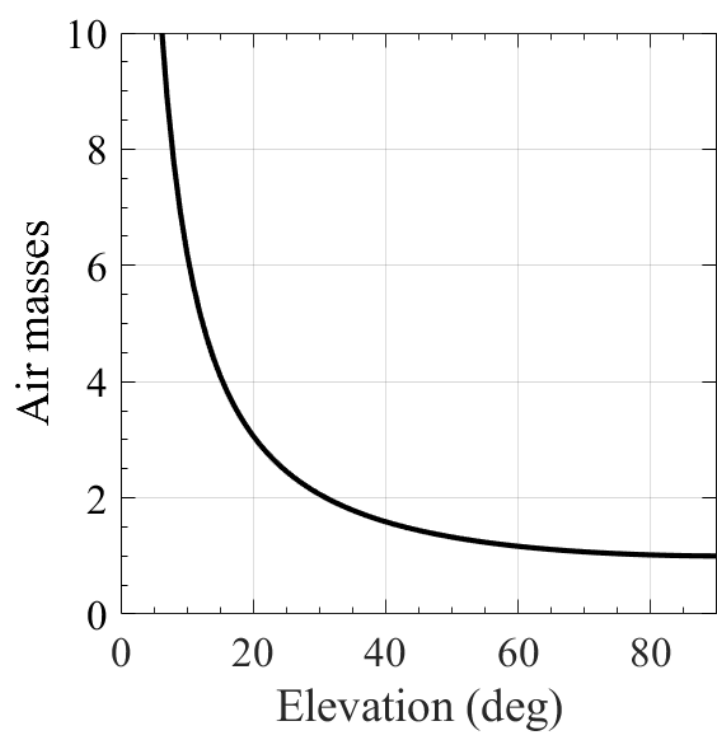

a)

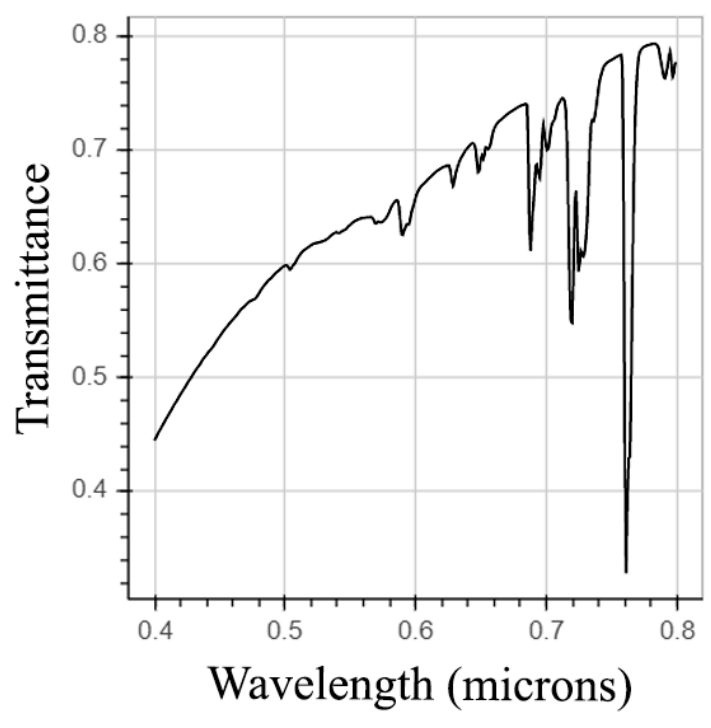

b)

Figure 3: Atmospheric parameters: Air masses versus elevation (plot a) and atmospheric transmittance versus wavelength (plot $b$, acquired from [33]).

The described optical link budget outputs a power at ground that must be collected by a receiving "antenna", which consists, in the analyzed case, in a telescope. The signal is then converted by a receiving sensor. Therefore, the telescope aperture and focal length are the key parameters to verify the detectability of the LEDs. The aperture quantifies the energy collected by the telescope while the focal length describes the field of view of the telescope and therefore the background noise collected. Then the sensor converts the light collected into a signal to be analyzed afterwards. Sensors are all photodiodes characterized by different data acquisition techniques. In particular the two main typologies are the arrayed (CCD and CMOS) and the single elements (APD and PIN) photodiodes.

The optical link on LEDSAT is established by matching the LEDs features with different ground segment operative modes, such as sidereal tracking and target tracking, as exposed in paragraph 2.2. The sensors used to detect the CubeSat are CCD and CMOS for the fixed mode and CMOS, APD and PIN photodiode for the tracking mode. Imaging sensors used in tracking mode take pictures with a high frame rate, while longer exposure pictures are taken during fixed mode to catch the passage of LEDSAT in the field of view. APD and PIN photodiodes are used only in tracking mode, by converting the optical signal into a voltage signal. 
To evaluate the feasibility of the optical link, two study cases has been analyzed, one for an ISS-like orbit and one for a SSO orbit of $700 \mathrm{~km}$. The slant range for the two orbits are shown in Figure 4.

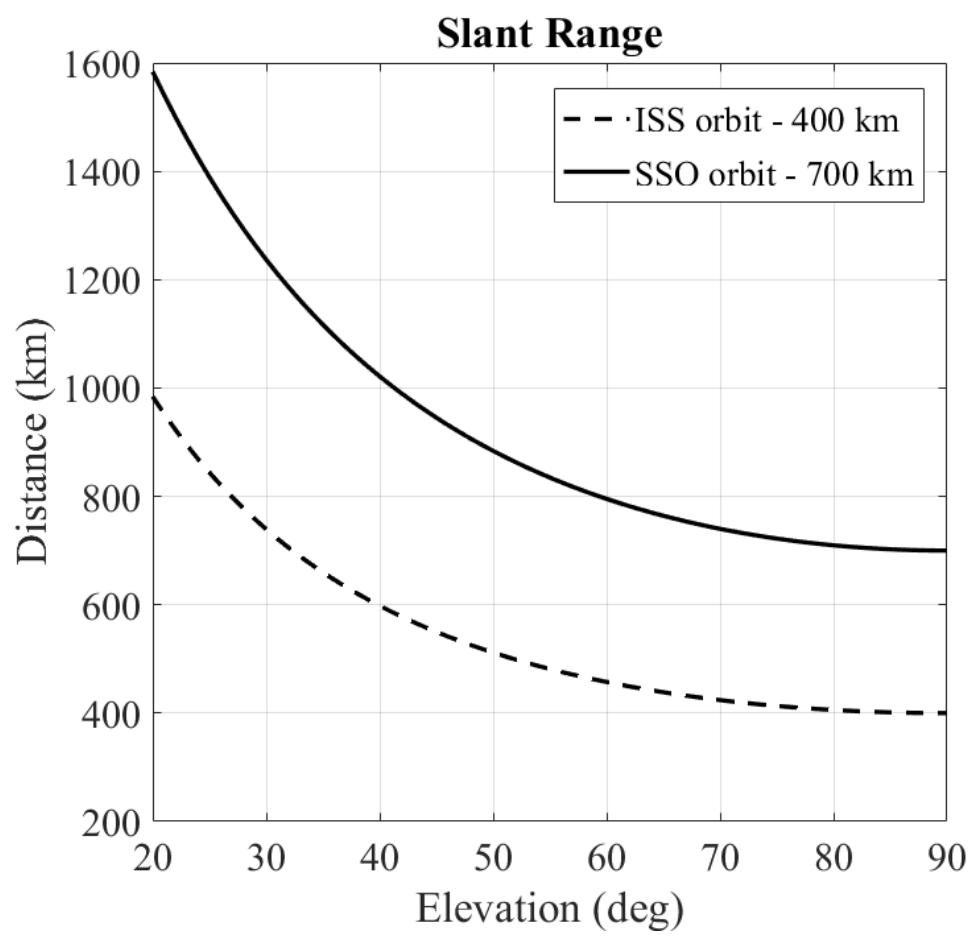

Figure 4: Slant range of a satellite on ISS orbit and SSO (700 km, 10.30 LTAN) orbit at different elevation angles.

The minimum elevation of 20 degrees is taken as a guideline because of the inverse exponential growth of the Air masses and Slant Range with the elevation (as also visible in Figure 3a). Below this threshold, the atmospheric extinction and the Free Space Path Loss became very high, causing a steep decrease of the SNR. In addition, the majority of astronomical telescopes cannot point below 15 degrees elevation. Therefore, this value is a useful reference for optical observations.

The apparent magnitude is the most common value used in astronomy to describe the brightness of an object in the sky as seen by an observer on Earth, therefore it is a simple way to evaluate the visibility of the targets with the sky as background. The apparent magnitude as a function of elevation angle for both of the considered orbits is shown in Figure 5. 


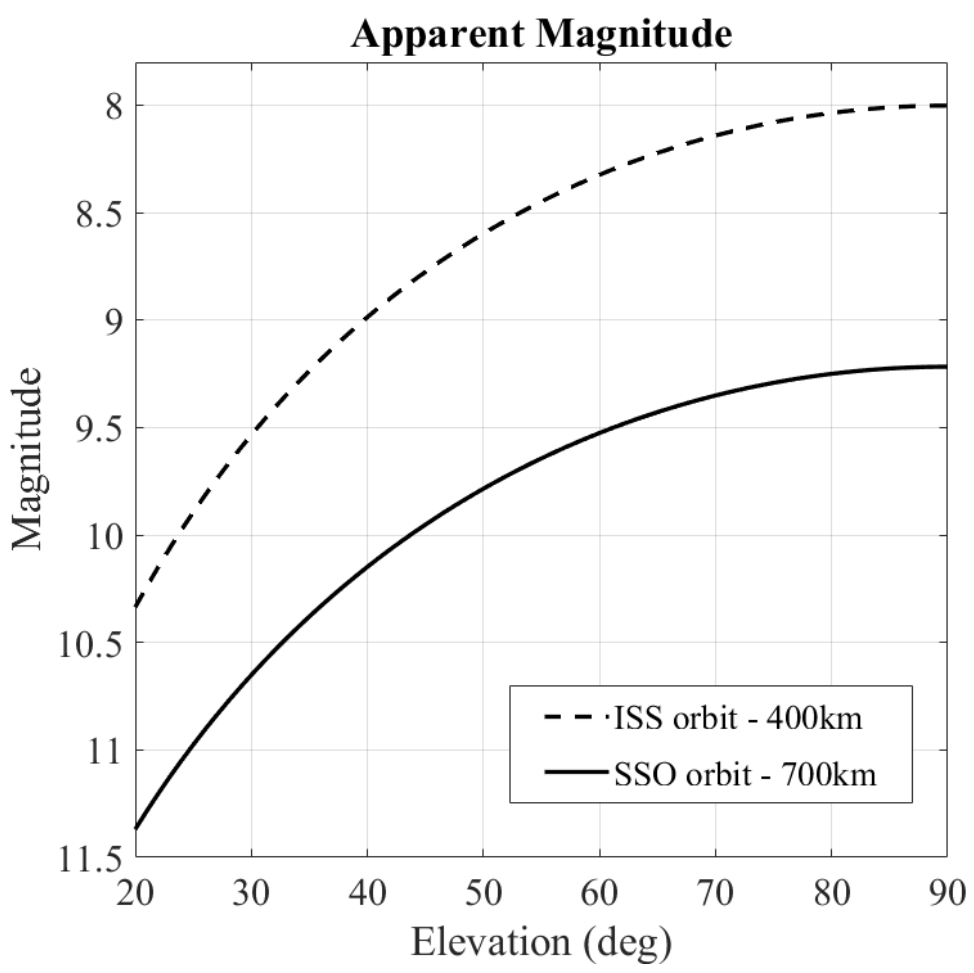

Figure 5: Apparent magnitude of the LED payloads.

Both study cases considered a SCMOS (scientific CMOS) sensor in sidereal and target tracking modes. A sCMOS sensor has been selected has a trade-off between the sensitivity of a CCD and the velocity of a single element photodiode. In sidereal tracking mode the SNR is limited by the satellite time on a pixel, while in target tracking mode it is limited by the frame per second related to the exposure time. The minimum time on pixel in relation with the pixels binning is shown in Figure 6 

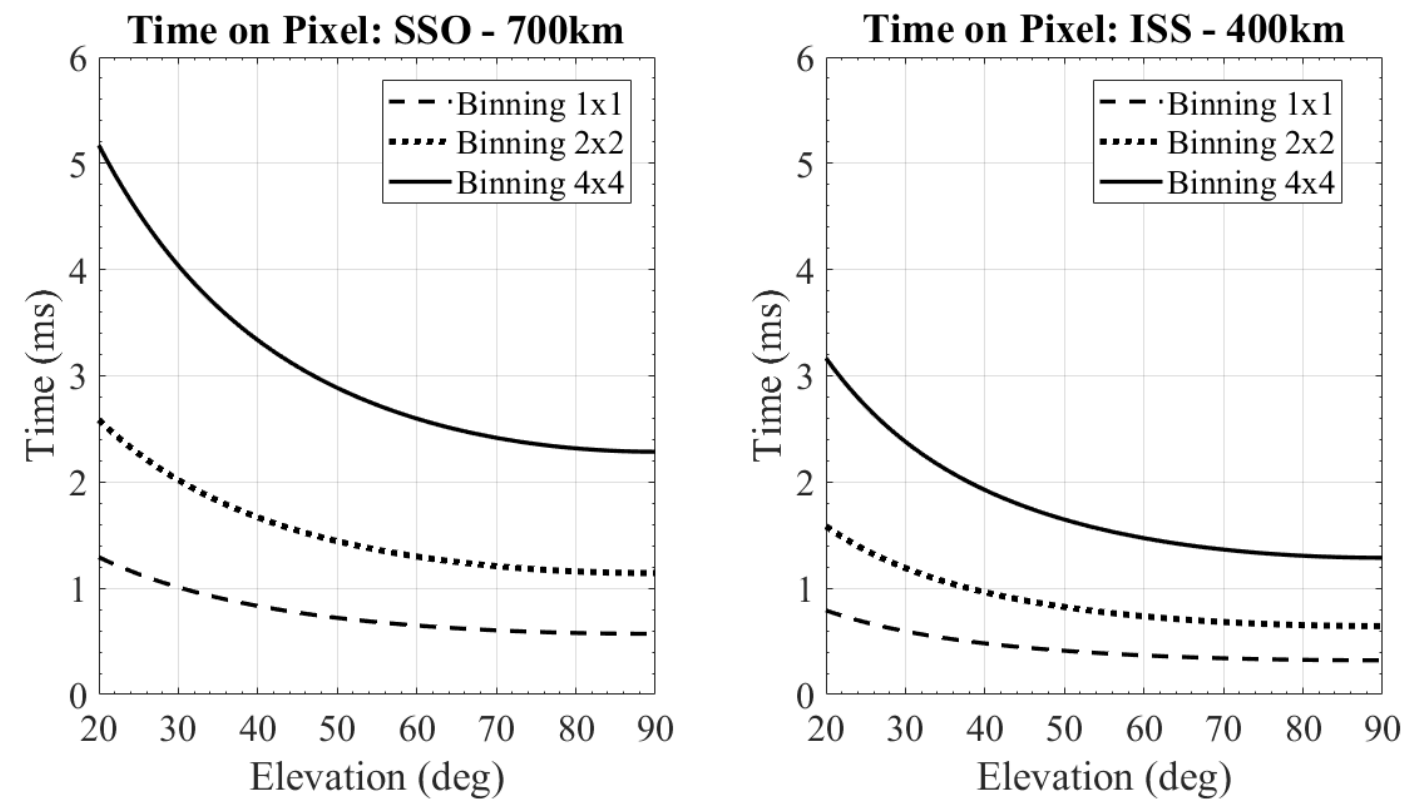

Figure 6: Time on Pixel of the spacecraft for the considered SSO and ISS orbits.

In the sidereal tracking mode, the SNR is limited by the permanence time of the satellite on a single pixel during the acquisition of a long exposure picture. The satellite angular velocity during a pass depends on the orbital parameters, mainly on the orbit altitude. It is obvious to derive that a satellite on a higher orbit will present a higher permanence time on a pixel. Moreover, the angular resolution of the telescope is related to the horizontal field of view. While, for an Earth Observation sensor, the spacecraft orbit directly influences the ground resolution of the on-board sensors, in the case of ground-based telescopes the target altitude defines the fraction of the orbit that is depictable in the FOV. Therefore, while for a lower orbit a satellite presents a higher orbital speed and a smaller portion of the orbit acquired in the sensor's FOV, a satellite on a higher orbit presents a lower speed and a larger fraction depictable in the FOV.

The pixel binning represents an advantageous tool as it gives the chance to create an artifact larger pixel by summing the acquired flux on adjacent pixels together. This leads to an increase of the satellite time on pixel and, consequently, of the acquisition SNR.

The SNR is evaluated by taking into consideration the background noise in the field of view, the shot noise, the dark current noise and the read noise. The target tracking mode exposure time is directly related to the data rate obtainable by the data link, as it is the sampling of the LED flashes. Therefore, the maximum data rate in tracking mode is half the sampling rate. The exposure times evaluated are $10 \mathrm{~ms}, 20 \mathrm{~ms}$ and $50 \mathrm{~ms}$. The resulting SNR as a function of elevation for sidereal and target tracking mode are shown in Figure 7 , Figure 8, Figure 9 and Figure 10. 


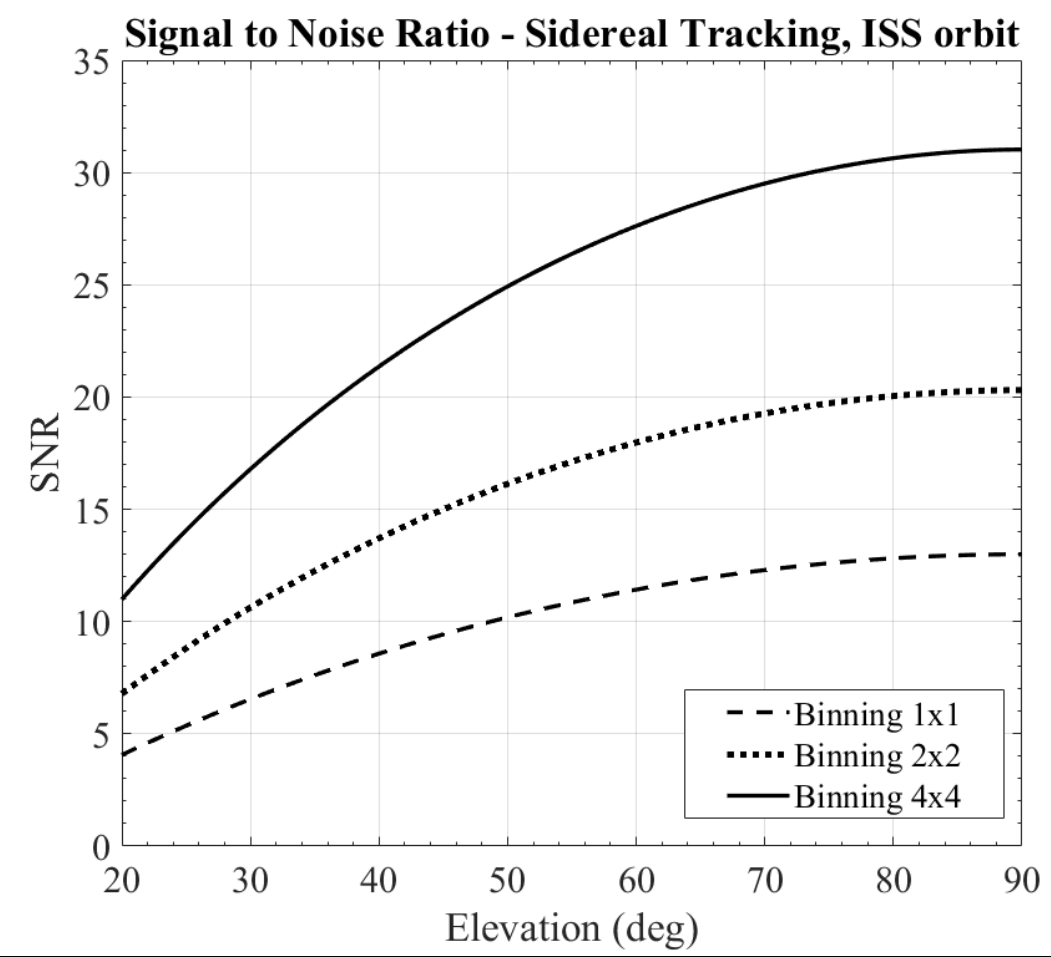

Figure 7: SNR in sidereal tracking mode, ISS orbit

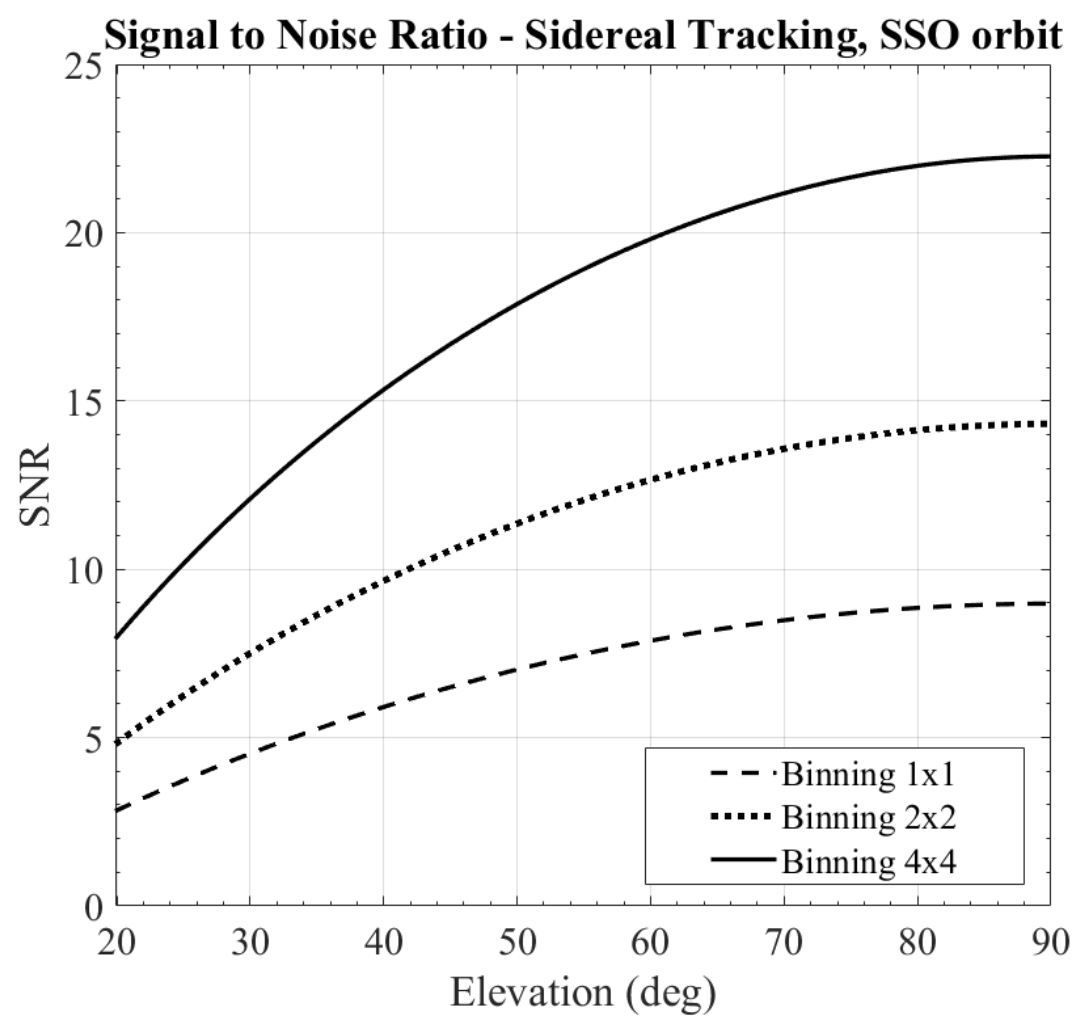

Figure 8: SNR in sidereal tracking mode, SSO (700 km) orbit 


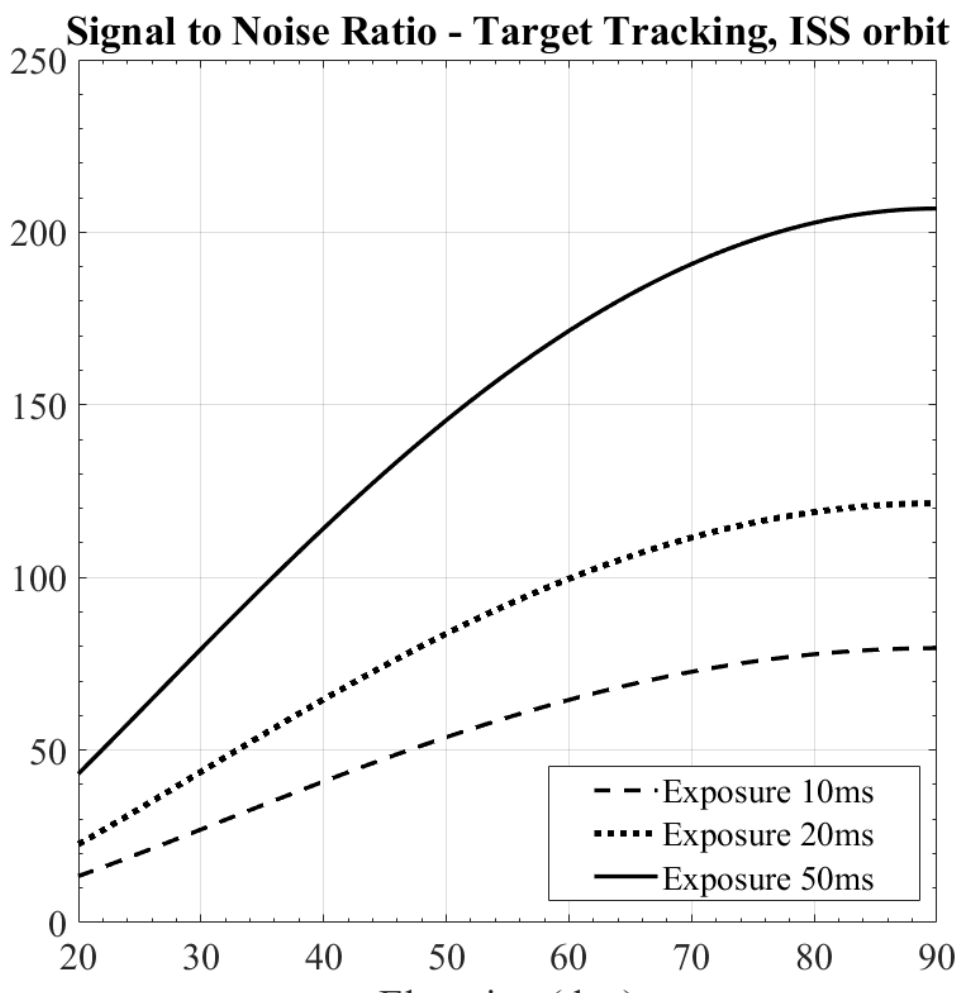

Elevation (deg)

Figure 9: SNR in target tracking mode, ISS orbit

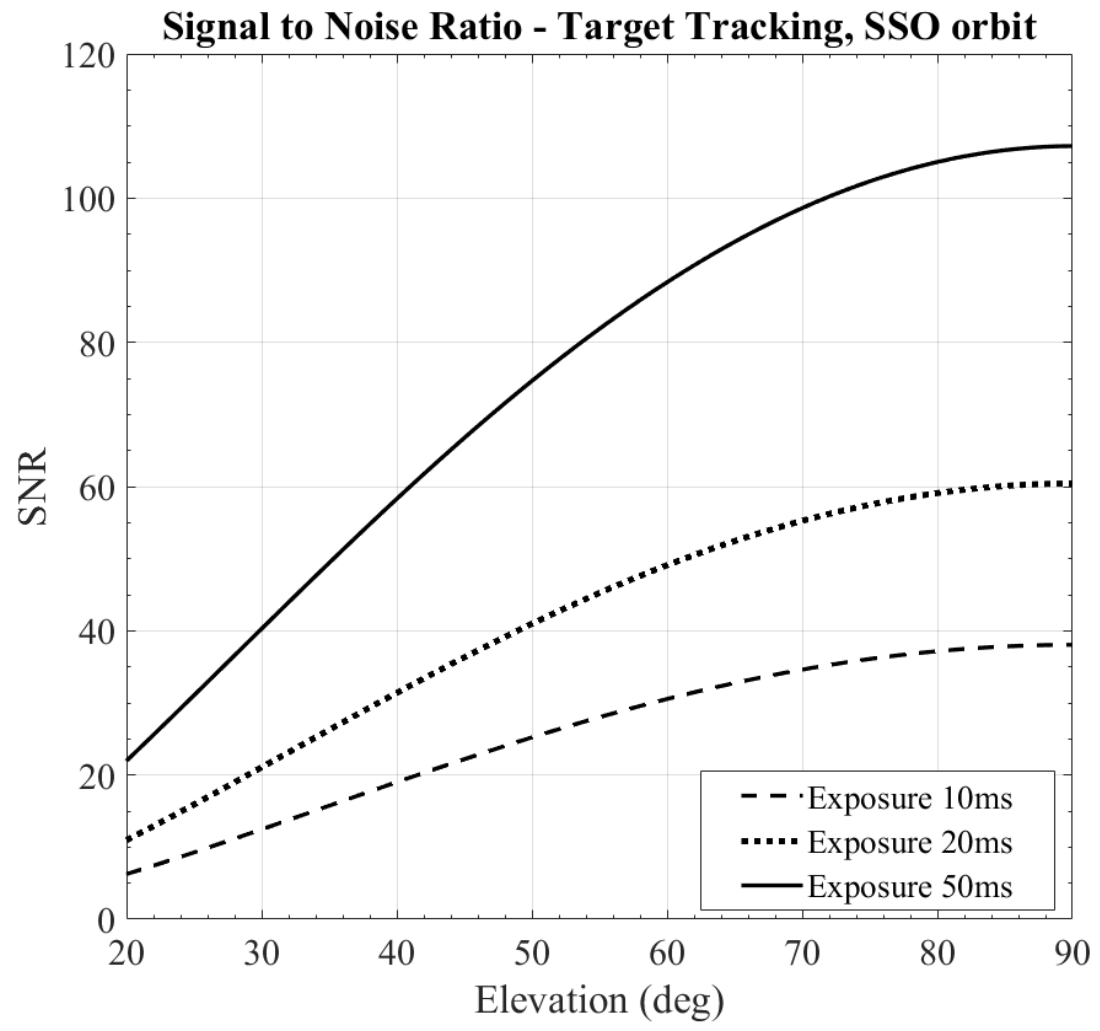

Figure 10: SNR in target tracking mode, SSO orbit 
The SNR results are positive for both modes and are higher for the tracking mode as expected. In order to enhance the stability of the link a minimum SNR of 10 is required. Experimental results on optical observations show how objects can be detected with a SNR as low as $2[5,7,31,34]$. However, a SNR of 10 is taken as a reference value for optical observation with commonly used astrometric software, as from the observation campaigns experience $[31,34]$ this value allows a good detection probability with a low chance of missing the detection due to noise. Below this threshold the probability of detection is significantly reduced. Moreover, the atmospheric conditions and the ground station set up and location may lead to a variation on the Noise parameters used in the analysis. Therefore, by considering the low exposure time of the targeted observations, the minimum SNR value of 10 is addressed as a conservative guideline or a best practice for optical observation of space objects for photometry and attitude reconstruction, as also shown in [35].

The results show that a higher binning may be required to maintain the minimum SNR level in sidereal tracking mode, while in target tracking mode the minimum SNR is achieved almost at all elevation with all exposure times. A summary of the results is shown in Table 4.

Table 4: SNR vs elevation analysis.

\begin{tabular}{|c|c|c|c|}
\hline \multicolumn{2}{|c|}{ Study case } & $\begin{array}{c}\text { Minimum SNR at } 20 \text { deg } \\
\text { elevation }\end{array}$ & $\begin{array}{c}\text { Minimum elevation for } \\
\text { SNR }=10\end{array}$ \\
\hline \multirow{3}{*}{$\begin{array}{c}\text { Sidereal tracking ISS } \\
\text { orbit }\end{array}$} & Binning $1 \times 1$ & 4 & $50 \mathrm{deg}$ \\
\hline & Binning $2 \times 2$ & 6.8 & 29 deg \\
\hline & Binning $4 \times 4$ & 11 & 19 deg \\
\hline \multirow{3}{*}{$\begin{array}{c}\text { Sidereal tracking SSO } \\
\text { orbit }\end{array}$} & Binning $1 \times 1$ & 3 & - \\
\hline & Binning $2 \times 2$ & 5 & $42 \mathrm{deg}$ \\
\hline & Binning $4 \times 4$ & 8 & $25 \mathrm{deg}$ \\
\hline \multirow{3}{*}{ Target tracking ISS orbit } & Exposure $10 \mathrm{~ms}$ & 13.5 & $17 \mathrm{deg}$ \\
\hline & Exposure $20 \mathrm{~ms}$ & 22.7 & 14 deg \\
\hline & Exposure $50 \mathrm{~ms}$ & 43 & $10 \mathrm{deg}$ \\
\hline \multirow{3}{*}{$\begin{array}{c}\text { Target tracking SSO } \\
\text { orbit }\end{array}$} & Exposure $10 \mathrm{~ms}$ & 6 & $27 \mathrm{deg}$ \\
\hline & Exposure $20 \mathrm{~ms}$ & 11 & $19 \mathrm{deg}$ \\
\hline & Exposure $50 \mathrm{~ms}$ & 22 & $13 \mathrm{deg}$ \\
\hline
\end{tabular}

\section{LED-based tracking operations}

As reported in the Introduction section, the LED payloads can be used during all the nano-satellite mission phases. During commissioning and LEOP, the flashes can enable the spacecraft recognition upon deployment, especially if the satellite has been deployed in a large cluster. During the nominal operations, the LED flashes can improve the orbit determination precision, when allowing the celestial coordinates acquisition and refinement, and the attitude determination accuracy, when attempting to recognize different patterns assigned to LED payloads on different faces. If some malfunctioning occurs to the telemetry link, the LEDs can serve as back-up, low data rate data transmission system, covering a key task of a spacecraft safe mode. Finally, the satellite Post-Mission Disposal (PMD) can take advantage of the LEDs for a faster passivation of the batteries, by enabling the LED payloads with a higher duty cycle to decrease the spacecraft electric energy storage. .. The following sub-paragraphs provide a more detailed insight on the functionalities and tasks of the LED boards Each paragraph will address a mission phase or satellite 
operative mode. For every operative mode, a practical example from the LEDSAT planned operations will be provided.

\subsection{Spacecraft early recognition}

Identifier LED sequences could be executed by the satellite soon after release and during LEOP. This feature would be of critical importance when satellites are deployed in large clusters. In this scenario, scarcely accurate TLEs maybe could lead to the confusion of a satellite with another of the same cluster. The misidentification can obstruct the observability of the satellite parameters during the first phase of the mission, impeding the communication with the satellite for days or weeks and to perform potentially mission saving maneuvers on time. The identification of satellites can be done by simply pre-programming the LED boards to flash with specifically assigned identifier flash sequences, by verifying a low crosscorrelation among all the sequences utilized by the satellites in the same cluster. For example, different Morse letters can be applied and observed $[8,10]$, or orthogonal patterns [36]. The identification task is mainly addressed at the sidereal rate observation technique, as the satellite TLE will be known with scarce precision and the tasks are addressed at a more precise target trajectory prediction.

In the case of LEDSAT, identification sequences will be performed soon after deployment [10]. Since the satellite will be probably launched in a small group (as per the usual procedures of the ISS deployment) and without other satellites implementing LEDs, the effectiveness of this technique will be verified by acknowledging the effective improvement introduced by the optical observations soon after deployment. The identification sequences will be optimized to be easily identifiable through an alternance of long and short flashes. The long flashes will be useful to immediately identify the satellite on the telescope images, while short flashes will be used for reconstructing the celestial coordinates of the satellite by identifying its relative position on the image and the background stellar field.

\subsection{Orbit Determination}

The analysis of the LED flashes over the background stellar field can improve a satellite orbit determination. In order to maximize the data return from the LED sequences observations, it is needed to optimize the flashes duration. Ideally, as per the spacecraft recognition tasks described in paragraph 3.1, as a general rule, flashing sequences should implement both long and short flashes sequences, i.e. in order to appear as long streaks and dots on the sensor image. The flash length shall be calculated by intersecting the orbital parameters of the satellite with the sensor characteristics. While long flashes are addressed at immediately identifying the satellite presence on a telescope image, either if the analysis is performed with a software or by hand, the short flashes can be used to immediately reconstruct the spacecraft orbit. As per paragraph 3.1, Morse code letters including both dots and dashes [8] can be applied. In this case, the LEDs optimization shall be addressed more at an improvement of the acquirable data and of their precision than on the discrimination of a known pattern. The orbit determination and refinement can benefit of the sidereal tracking technique, in order to apply to the acquired flashes the available astrometric methodologies for space debris optical orbit determination [31].

LEDSAT will use both Morse letters and orthogonal codes (that will be presented in the attitude determination feature in paragraph 3.3) for the orbit determination. As already reported, the preferred observational technique for achieving an improvement in orbit determination is the sidereal tracking technique, by means of an imaging sensor. 


\subsection{Attitude reconstruction}

Optical attitude determination with LED payloads can be maximized when considering multiple LED boards on different sides of the satellite. If the LEDs are implemented in different colors (e.g. with a green board on one side and one red board on another side), the attitude reconstruction can be carried out by means of simultaneous observations from multiple sites implementing color filters. A more precise attitude determination can be achieved by flashing the LED with different patterns, making it possible to observe the satellite and to recognize which satellite side is pointing towards the observation station. The application or design of orthogonal patterns can allow the flashing sequences to present a low crosscorrelation and a high self-correlation. The most convenient way to extrapolate information from this method is to perform target tracking observations with a CMOS sensor shooting a high framerate video to the satellite passage. In this way, videos of several minutes can be processed in order to access a higher quantity of data.

In the LEDSAT case, LED boards are on all the surfaces, with opposite faces equipping the same diodes color. The LED patterns for attitude determination have been derived from the Gold codes (used for the GPS Pseudo-Random Number, PRN, codes transmitted both in the Coarse Acquisition and in the Precision codes for accurate positioning [36]). These patterns allow to recognize the face pointing towards the observer with even a low percentage of the flashes observed and acquired by the sensor. The satellite will use relatively long $(0.5 \mathrm{~s})$ flashes in order to maximize the chances of being recognized both in videos and images. Simulations performed to identify the usefulness and effectiveness of the method have showed perfect compliance with the LEDSAT mission objectives.

\subsection{Light Communication}

The LEDs can be commanded to downlink basic data at low rate to ground. Different encoding methods can be used for the data downlink. The achievable data rate varies in relation to the operative modes and the sensor used. For the simpler methods, such as the fixed mode with imaging sensors, a very low data rate can be achieved as the flashing LEDs appear as dots and lines on a long exposure picture. The link capacity is therefore limited by the time of passage of the spacecraft through the field of view. For the tracking mode CMOS and single element sensors are used. In this case the link time is extended to the whole passage of the CubeSat over the ground station. The utilization of a CMOS imaging sensor considers a high frame rate video to be taken to detect the binary flashes of the LEDs, since the data rate is proportional to the frame rate of the video that correspond to the sampling rate of the signal. Single element sensors convert directly light into a voltage signal, so a real time link can be performed, and have a higher sampling rate therefore a higher data rate can be achieved. The main drawback is the higher background noise that they collect from all the field of view of the telescope, so a high focal length telescope should be used.

When considering an exposure time of $10 \mathrm{~ms}$ in target tracking mode, corresponding to a sampling rate of $100 \mathrm{fps}$, the maximum achievable data rate is 50 bit per second. For an average passage over a mid-latitude ground the mean access time and the data volume per passage is shown in Table 5. 
Table 5: Mean access time over a mid-latitude ground station

\begin{tabular}{ccc}
\hline Orbit & $\begin{array}{c}\text { Mean access } \\
\text { time }\end{array}$ & $\begin{array}{c}\text { Data volume } \\
\text { per passage }\end{array}$ \\
ISS $-400 \mathrm{~km}$ & $570 \mathrm{~s}$ & $28.5 \mathrm{kbit}$ \\
SSO $-700 \mathrm{~km}$ & $660 \mathrm{~s}$ & $33 \mathrm{kbit}$ \\
\hline
\end{tabular}

The experimental mode of the LEDSAT CubeSat will test the downlink of dummy and housekeeping data through LEDs. In preparation for the mission, several long range data transmission tests have been performed on the GS equipment. The PIN photodiode has been tested on an $80 \mathrm{~mm}$ telescope at $400 \mathrm{~m}$ distance in Anagni (Italy), while a COTS CMOS Camera has been tested on a $250 \mathrm{~mm}$ telescope at a distance of around $9 \mathrm{~km}$ over the main diameter of lake of Bracciano (Italy). The set-up on the lakeshore is shown in Figure 11[37]. In both tests a light signal has been transmitted by the LEDs board, received, converted to voltage and analyzed. While the first long range test demonstrated the functionalities of the ground system and of the photodiode, by receiving and decoding a K-Morse letter (as in Figure 12[37]), the Bracciano lake long range test consisted in transmitting binary data packets to be sampled by CMOS camera in sequential pictures allowing the 3D visualization of the signal into the pixels array is shown in Figure 13 [37]. The signal level shows a similar result to the link budget calculation in a very worst case for atmospheric absorption which is compensated by the shorter distance than the case of a satellite.

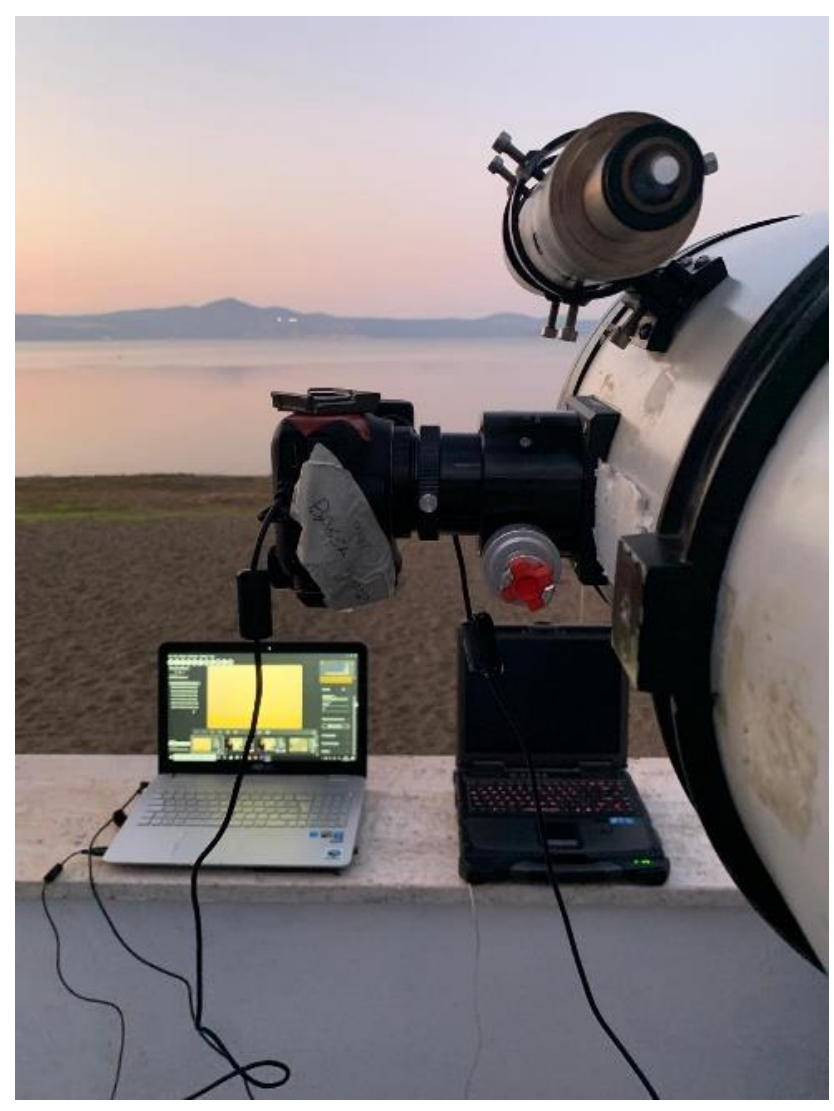

Figure 11: Test set-up at Lake Bracciano, Italy, September 2019 [37] 


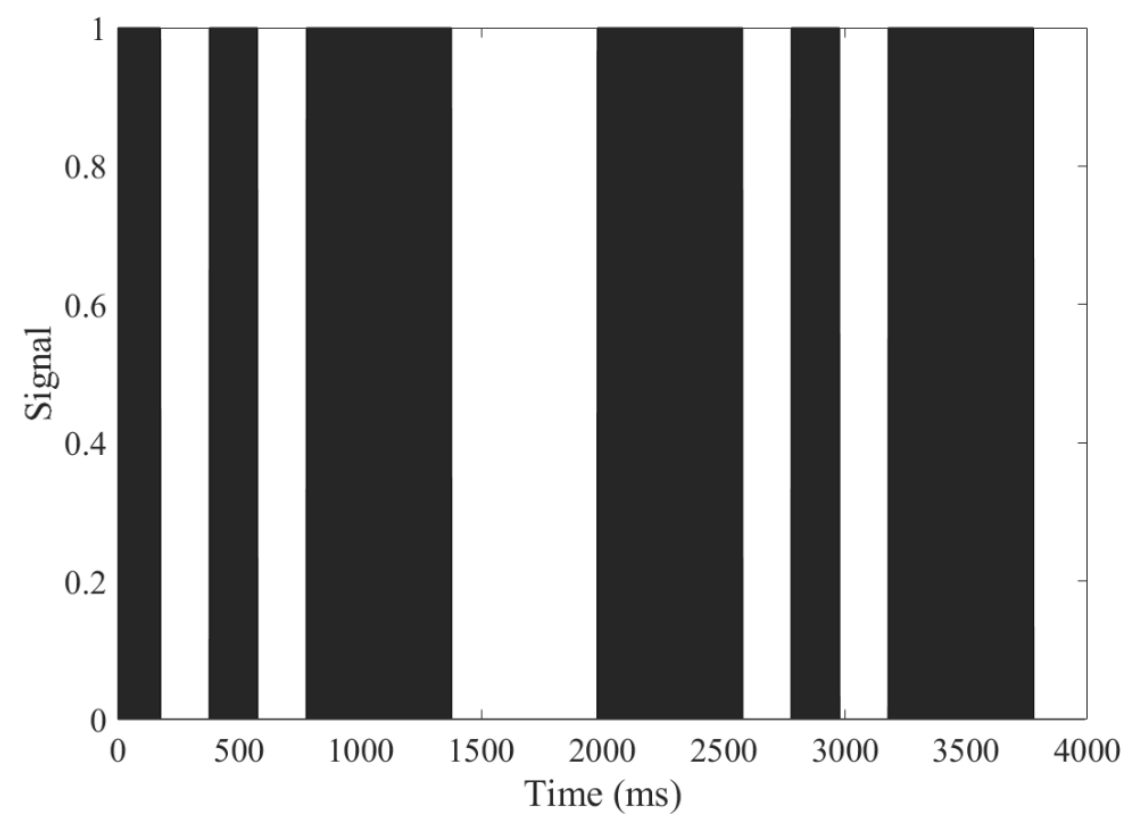

Figure 12: Decoded K-Morse letter [37]

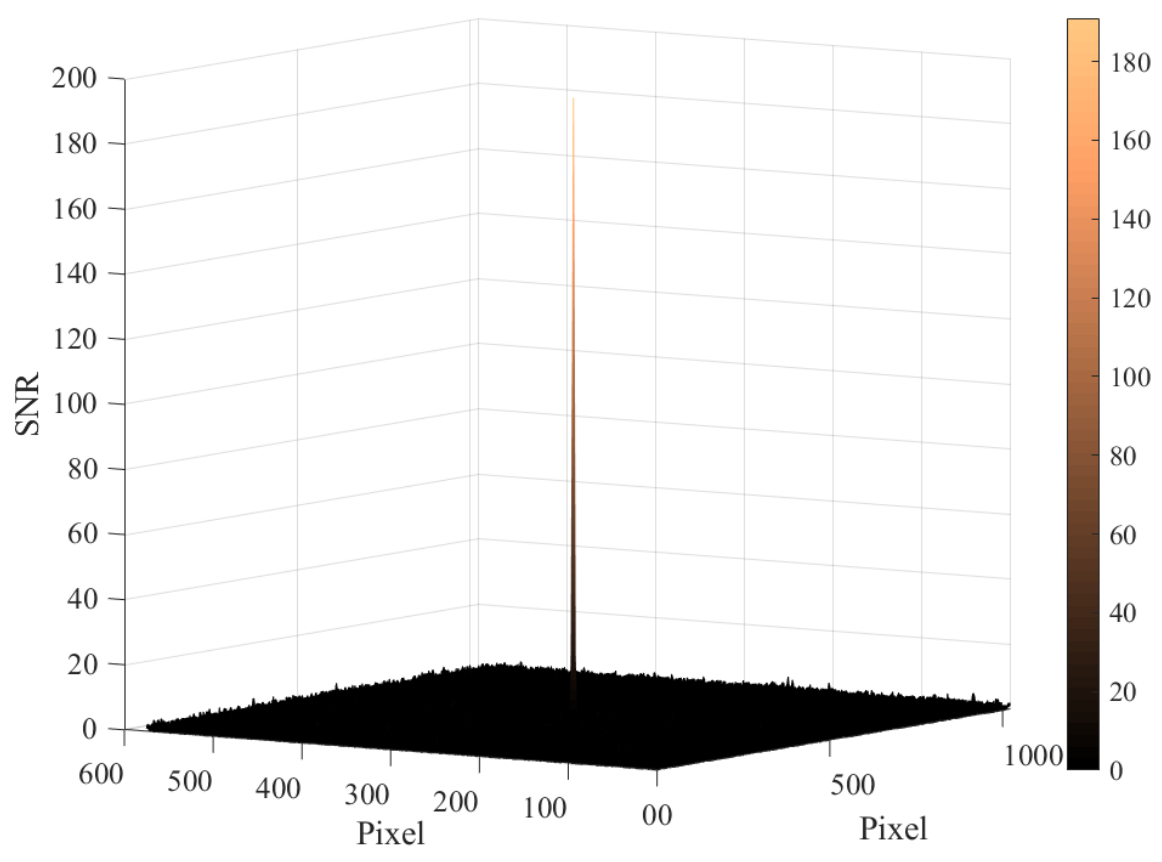

Figure 13: Signal level in the pixels array [37]

\subsection{Post Mission Disposal (PMD)}

The LEDs can be triggered to support the Post Mission Disposal (PMD) phase of the mission. After the mission conclusion, it is of paramount importance to ensure that the satellite will re-entry the atmosphere within a reasonable time and to diminish the storage of all types of energy [38]. While chemical energy in propulsion systems can be depleted by venting the propellant tanks or by performing a perigee reduction maneuver, the batteries state of charge needs to be reduced. This can be done by physically disconnecting 
the batteries from the solar panels and by activating the payloads that can consume energy without threatening or disturbing the in-orbit activities of other satellites. In this perspective, the LEDs can be activated with a higher duty cycle at the end of the mission, leading to a significant reduction of the battery charge.

\section{Conclusions}

The increasingly frequent launches of satellite constellation and clusters, with particular attention to small satellite platforms, require the actually operative tracking systems to be more precise and responsive in the perspective of future Space Situational Awareness and Space Traffic Management tasks.

If implementing active illumination payloads such as Light-Emitting Diodes-based boards, satellites are able to be optically tracked throughout the eclipse phase, thus without constraint related to light conditions and Sun phase angle.

LED payloads can support the tracking for the entire orbital lifetime of a satellite, from early recognition upon deployment in a large cluster to orbit determination, attitude reconstruction, back-up light communication and support to Post-Mission Disposal tasks.

The design of a LED-based optical tracking system should take into account the maximization of the irradiance, as allowed by power and available volume constraints, in order to involve small telescope stations in the ground segment. The spaceborne LED-based system can consider between 15 and 40 diodes (by maximizing Quantum Efficiency and irradiance as possible) if implementing commercial LEDs, respecting the maximum allowable power constraints of nano-satellite platforms electrical power systems. The diodes could execute identifier patterns for recognition and orbit determination every 25-30 seconds by consuming a small portion (5\%) of the energy generated by a reference $1 U$ CubeSat EPS. This energy consumption makes the LED system compatible with the most constrained CubeSat platform, a $1 \mathrm{U}$ satellite, and with the implementation of LEDs as secondary tracking system.

The ground segment can consider telescopes with relatively low-cost hardware. The observational techniques are considering the telescopes to move with sidereal rate, acquiring the satellite LED signal as a strike over the stellar background, or tracking the target and maintaining it in the FOV for the whole pass. Both arrayed (CCD and CMOS) and single element (P-I-N or APD photodiodes) sensors can be applied for different tasks and with different advantages and drawbacks. While photodiodes acquisition can allow to extrapolate flight data from the flashes execution, CCD or CMOS based techniques are able to provide tracking and attitude information.

When calculating the optical link budget, the most constraining parameters to take into account are the minimum elevation and the atmospheric transmittance at the LEDs flashing wavelength, in addition to the ground segment features, e.g. the sensor's features and the selected observation technique. As a guideline, a minimum SNR of 10 and a minimum elevation of 20 degrees can be taken as guidelines for the data acquisition from ground.

While the flashing patterns can be optimized for multiple purposes, the most basic guideline for LED flashes is to take into account both long and short flashes in order both to identify the satellite over the background stellar field and to reconstruct the target celestial coordinates. Orthogonal patterns, e.g. Gold codes, can improve the recognition of different patterns executed at the same time, as for attitude determination through discrimination of the different satellites sides flashing with different patterns. The 
implementation of LEDs in different colors can provide a further advantage when allowing simultaneous observations with color filters from multiple sensors and/or sites.

The LEDSAT 1U CubeSat has been used as study case for this paper. The satellite will investigate the performances of a LED-based payload by implementing LEDs in three different bandwidths on all the satellite's sides. The spacecraft will test early recognition, orbit determination, attitude determination and back-up light-based communication tasks during its mission. The satellite launch is currently scheduled for Q1 2021.

\section{Acknowledgements}

The LEDSAT project is supported by the Italian Space Agency, which the authors gratefully acknowledge, in the framework of the IKUNS (Italian-Kenyan University Nano-Satellites) Programme (Accordo IKUNSItalian/Kenyan University Nanosatellites - n.2015-031R.1-2018).

LEDSAT is part of the second edition of the Fly Your Satellite! Educational Programme, managed by the Education Office of the European Space Agency (ESA). Within the Programme, students design and build their satellite at their universities and benefit from direct knowledge transfer of ESA technical and managerial expertise, as well as access to facilities.

As referred before, the project is supported by the Astronomy Institute of the University of Bern (AIUB) and by the ASI Center of Space Geodesy (ASI CGS), since the observational campaigns and the laser ranging measures will be performed by these institutions.

The authors wish to thank Profilocolore Srl, which for offering the needed equipment for the radiometric measurements campaigns, and NPC (New Production Concept) Srl, for providing support and for designing and producing the CubeSat structural system.

\section{References}

[1] D. McKnight, A practical perspective on Space Traffic Management, J. Space Saf. Eng. 6 (2019) 101107. https://doi.org/10.1016/j.jsse.2019.03.001.

[2] M.A. Skinner, M.K. Jah, D. McKnight, D. Howard, D. Murakami, K.-U. Schrogl, Results of the international association for the advancement of space safety space traffic management working group, J. Space Saf. Eng. 6 (2019) 88-91. https://doi.org/10.1016/j.jsse.2019.05.002.

[3] C. Bonnal, L. Francillout, M. Moury, U. Aniakou, J.-C. Dolado Perez, J. Mariez, S. Michel, CNES technical considerations on space traffic management, Acta Astronaut. 167 (2020) 296-301. https://doi.org/10.1016/j.actaastro.2019.11.023.

[4] M. Skinner, Making small satellites visible: Nanosat tracking and identification techniques and technologies (IAC-19-A6.7.5), in: Proc. 70th Int. Astronaut. Congr. IAC, 2019.

[5] F. Piergentili, A. Ceruti, F. Rizzitelli, T. Cardona, M.L. Battagliere, F. Santoni, Space Debris Measurement Using Joint Mid-Latitude and Equatorial Optical Observations, IEEE Trans. Aerosp. Electron. Syst. 50 (2014) 664-675. https://doi.org/10.1109/TAES.2013.120272.

[6] S. Hadji Hossein, F. Curiano, L. Mariani, M. Acernese, P. Marzioli, T. Cardona, V. Marini, L. Parisi, Sapienza Space Systems and Space Surveillance Network (S5N): a high coverage infrastructure for space debris monitoring., in: Bremen, Germany, 2018.

[7] F. Santoni, F. Piergentili, R. Kawashima, P. Marzioli, M. Acernese, GUSDON (Global University Students Observation Network): improvements on space debris optical monitoring offered by a global University network, in: Proc. 12th IAA Symp. Small Satell. Earth Obs., IAA, Berlin, Germany, 2019. http://smallsat.iaaweb.org/ (accessed February 13, 2020). 
[8] P. Seitzer, J. Cutler, F. Piergentili, F. Santoni, L. Arena, T. Cardona, H. Cowardin, C. Lee, S. Sharma, LEDsats: LEO Cubesats with LEDs for Optical Tracking, in: 2016.

[9] P. Seitzer, J.H. Schachter, M. Szczerba, D. Gu, A.X. Lin, C.H. Lee, J. Cutler, P. Washabaugh, N. Estell, G. Bryja, Optical Tracking and Attitude Determination of LEO CubeSats with LEDs: A Balloon Demonstration, AMOS Technol. Conf. Maui Hawaii USA. (2018).

[10] J. Cutler, P. Seitzer, C.H. Lee, P. Washabaugh, S. Sharma, R. Gitten, F. Piergentili, F. Santoni, T. Cardona, G. Cialone, L. Frezza, A. Gianfermo, P. Marzioli, S. Masillo, A. Pellegrino, T. Schildknecht, Improved Orbit Determination of LEO CubeSats: Project LEDsat, in: Maui, Hawai'i, USA, 2017.

[11] P. Marzioli, A. Pellegrino, G. Cialone, A. Gianfermo, L. Frezza, S. Masillo, F. Curiano, A. Grossi, P. Seitzer, S. Pirrotta, F. Piergentili, F. Santoni, Opportunities and technical challenges offered by a LEDbased technology on-board a CubeSat: The LEDSAT mission, in: Proc. 69th Int. Astronaut. Congr., 2018.

[12] M. Swartwout, The First One Hundred CubeSats: A Statistical Look, (2013) 213-233.

[13] A. Gianfermo, P. Marzioli, G. Cialone, F. Piergentili, F. Santoni, S. Pirrotta, P. Seitzer, LED-based optical communication on a nano-satellite platform, in: Proc. Int. Astronaut. Congr. IAC-18B274x47709 2018Oct., Bremen; Germany, n.d.

[14] X. Wang, M. Zhang, H. Zhou, X. Lin, X. Ren, A Robust real-time automatic recognition prototype for maritime optical morse-based communication employing modified clustering algorithm, Appl. Sci. Switz. 10 (2020). https://doi.org/10.3390/app10041227.

[15] M.A. Khalighi, M. Uysal, Survey on free space optical communication: A communication theory perspective, IEEE Commun. Surv. Tutor. 16 (2014) 2231-2258. https://doi.org/10.1109/COMST.2014.2329501.

[16] P. Du, S. Zhang, W.-D. Zhong, C. Chen, H. Yang, A. Alphones, R. Zhang, Real-time indoor positioning system for a smart workshop using white LEDs and a phase-difference-of-arrival approach, Opt. Eng. 58 (2019). https://doi.org/10.1117/1.OE.58.8.084112.

[17] P. Fiorentin, C. Bettanini, D. Bogoni, Calibration of an autonomous instrument for monitoring light pollution from drones, Sens. Switz. 19 (2019). https://doi.org/10.3390/s19235091.

[18] J. Rivas, J. Martinez-Oter, I. Arruego, A. Martin-Ortega, J.R. De Mingo, J.J. Jimenez, B. Martin, Owls as platform technology in OPTOS satellite, in: 2017. https://doi.org/10.1117/12.2296165.

[19] J.J. Jiménez, M.T. Alvarez, R. Tamayo, J.M. Oter, J.A. Domínguez, I. Arruego, J. Sánchez-Páramo, H. Guerrero, Proton radiation effects in high power LEDs and IREDs for optical wireless links for intrasatellite communications (OWLS), in: 2006: pp. 77-84. https://doi.org/10.1109/REDW.2006.295472.

[20] D.N. Amanor, W.W. Edmonson, F. Afghah, Intersatellite Communication System Based on Visible Light, IEEE Trans. Aerosp. Electron. Syst. 54 (2018) 2888-2899. https://doi.org/10.1109/TAES.2018.2832938.

[21] A. Nakajima, N. Sako, M. Kamemura, Y. Wakayama, A. Fuzukawa, H. Sugiyama, N. Okada, ShindaiSat : A Visible Light Communication Experimental Micro-Satellite, in: Proc. Int. Conf. Space Opt. Syst. Appl. ICSOS 2012, Ajaccio, Corsica, France, 2012.

[22] T. Tolker-Nielsen, G. Oppenhaeuser, In orbit test result of an operational optical intersatellite link between ARTEMIS and SPOT4, SILEX, in: 2002: pp. 1-15. https://doi.org/10.1117/12.464105.

[23] D.M. Boroson, B.S. Robinson, D.V. Murphy, D.A. Burianek, F. Khatri, J.M. Kovalik, Z. Sodnik, D.M. Cornwell, Overview and results of the lunar laser communication demonstration, in: 2014. https://doi.org/10.1117/12.2045508.

[24] Z. Sodnik, B. Furch, H. Lutz, Optical intersatellite communication, IEEE J. Sel. Top. Quantum Electron. 16 (2010) 1051-1057. https://doi.org/10.1109/JSTQE.2010.2047383.

[25] P. Villoresi, T. Jennewein, F. Tamburini, M. Aspelmeyer, C. Bonato, R. Ursin, C. Pernechele, V. Luceri, G. Bianco, A. Zeilinger, C. Barbieri, Experimental verification of the feasibility of a quantum channel between space and Earth, New J. Phys. 10 (2008). https://doi.org/10.1088/1367-2630/10/3/033038.

[26] G. Vallone, D. Bacco, D. Dequal, S. Gaiarin, V. Luceri, G. Bianco, P. Villoresi, Experimental Satellite Quantum Communications, Phys. Rev. Lett. 115 (2015). https://doi.org/10.1103/PhysRevLett.115.040502. 
[27] S.-K. Liao, W.-Q. Cai, W.-Y. Liu, L. Zhang, Y. Li, J.-G. Ren, J. Yin, Q. Shen, Y. Cao, Z.-P. Li, F.-Z. Li, X.-W. Chen, L.-H. Sun, J.-J. Jia, J.-C. Wu, X.-J. Jiang, J.-F. Wang, Y.-M. Huang, Q. Wang, Y.-L. Zhou, L. Deng, T. Xi, L. Ma, T. Hu, Q. Zhang, Y.-A. Chen, N.-L. Liu, X.-B. Wang, Z.-C. Zhu, C.-Y. Lu, R. Shu, C.-Z. Peng, J.-Y. Wang, J.-W. Pan, Satellite-to-ground quantum key distribution, Nature. 549 (2017) 43-47. https://doi.org/10.1038/nature23655.

[28] G. Vallone, V. D’Ambrosio, A. Sponselli, S. Slussarenko, L. Marrucci, F. Sciarrino, P. Villoresi, Free-space quantum key distribution by rotation-invariant twisted photons, Phys. Rev. Lett. 113 (2014). https://doi.org/10.1103/PhysRevLett.113.060503.

[29] T. Tanaka, Y. Kawamura, T. Tanaka, Development and operations of nano-satellite FITSAT-1 (NIWAKA), Acta Astronaut. 107 (2015) 112-129. https://doi.org/10.1016/j.actaastro.2014.10.023.

[30] G. Cialone, P. Marzioli, S. Masillo, A. Gianfermo, L. Frezza, A. Pellegrino, F. Piergentili, F. Santoni, LEDSAT: a LED-Based CubeSat for Optical Orbit Determination Methodologies Improvement, in: 2018 5th IEEE Int. Workshop Metrol. Aerosp. MetroAeroSpace, 2018: pp. 456-461. https://doi.org/10.1109/MetroAeroSpace.2018.8453518.

[31] S. Hadji Hossein, M. Acernese, T. Cardona, G. Cialone, F. Curianò, L. Mariani, V. Marini, P. Marzioli, L. Parisi, F. Piergentili, F. Santoni, Sapienza Space debris Observatory Network (SSON): A high coverage infrastructure for space debris monitoring, J. Space Saf. Eng. (2019). https://doi.org/10.1016/j.jsse.2019.11.001.

[32] T. Cardona, F. Curianò, F. Piergentili, F. Santoni, M. Castronuovo, Scheduling Solution for Space Debris Observations, Adv. Astronaut. Sci. Technol. 1 (2018) 45-56. https://doi.org/10.1007/s42423-0180015-3.

[33] Spectral Sciences, Inc., MODTRAN (MODerate resolution atmospheric TRANsmission) computer code, Spectral Sciences, Inc. (SSI), n.d. http://modtran.spectral.com/modtran_home (accessed May 6, 2020).

[34] F. Piergentili, F. Santoni, P. Seitzer, Attitude Determination of Orbiting Objects from Lightcurve Measurements, IEEE Trans. Aerosp. Electron. Syst. PP (2017) 1-1. https://doi.org/10.1109/TAES.2017.2649240.

[35] J.R. Shell, Optimizing orbital debris monitoring with optical telescopes, in: Hawai'i, USA, 2010.

[36] P. Marzioli, A. Gianfermo, L. Frezza, D. Amadio, M. Acernese, L. Parisi, G. Cialone, M.G. Pancalli, E. Vestito, F. Curianò, N. Picci, F. Piergentili, F. Santoni, LED-based attitude reconstruction and back-up light communication: experimental applications for the LEDSAT CubeSat, in: 2019 IEEE 5th Int. Workshop Metrol. Aerosp. MetroAeroSpace, 2019: pp. 720-725. https://doi.org/10.1109/MetroAeroSpace.2019.8869673.

[37] A. Gianfermo, P. Marzioli, L. Frezza, D. Amadio, E. Vestito, M.G. Pancalli, S. Hdaji Hossein, L. Mariani, D. Gu, A. Lin, J.H. Schachter, M. Szczerba, F. Piergentili, F. Santoni, S. Pirrotta, J. Cutler, P. Seitzer, Development and Testing of a LED-based Optical Data Link for the LEDSAT CubeSat IAC19,B2,2,8,x53908, in: Proc. 70th Int. Astronaut. Congr. IAC, Washington D.C., 2019.

[38] International Academy of Astronautics (IAA, Study Group 4.23), A Handbook for Post-Mission Disposal of Satellites Less Than $100 \mathrm{~kg}$, (2019). https://iaaweb.org/iaa/Scientific\%20Activity/sg423finalreport.pdf (accessed April 23, 2020). 